\title{
Building the Road to a Regional Zoonoses Strategy: a Survey of Zoonoses Programs in the Americas
}

\author{
Melody J. Maxwell ${ }^{* 1,2}$, Mary Hofmeister Freire de Carvalho ${ }^{2}$ and Victor del rio vilas ${ }^{2}$ \\ 'The Ohio State University, Columbus, OH, USA; 2PANAFTOSA (PAHO/WHO), Rio de Janeiro, Brazil
}

\section{Objective}

To take the first step in identifying how American countries can strengthen their capacities to manage zoonoses risks by capturing information regarding their national zoonoses programs and priorities.

\section{Introduction}

Zoonoses account for over $70 \%$ of emerging infectious diseases in humans ${ }^{1}$. In recent years, global public health security has been threatened by zoonotic disease emergence as exemplified by outbreaks of H5N1 and H1N1 influenza, SARS, and most recently Ebola. The occurrence of a number of these zoonoses, and their spread to new areas, is related to globalization, environmental changes, and marginalization of populations. This phenomenon holds true for Latin American and the Caribbean countries (LAC), where $70 \%$ of the events public health emergencies reported to the WHO from 2007 to 2008 in the Americas were classified as zoonoses or communicable diseases common to humans and animals ${ }^{2}$. Despite this record, there are no national or regional disease burden estimates in LAC for many zoonoses ${ }^{3}$. To start filling this void, the Pan American Health Organization (PAHO) conducted a survey of LAC countries to collect information on priority emerging and endemic zoonoses, countries prioritization criteria and methodologies, and suggestions to strengthen countries capacities and regional approaches to zoonoses control.

\section{Methods}

An online questionnaire was sent to the zoonoses program managers of the Ministries of Health $(\mathrm{MOH})$ and Ministries of Agriculture (MAg) of all 33 LAC countries from January to April of 2015. The questionnaire comprised 36 single, multiple choice and open-ended questions to inform the objectives of the survey. A descriptive exploratory analysis was completed in R (i386 3.1.2).

\section{Results}

Fifty-four ministries (26 MOH, $25 \mathrm{MAg}$, and 3 combined responses) in $31 \mathrm{LAC}$ countries responded to the survey. Within the ministries, 22 (85\%) MOH, 5 (20\%) MAg, and 2 (67\%) combined entities indicated they had specialized zoonoses units.

For endemic zoonoses, 32 ministries responded that they conduct formal prioritization exercises, most of them annually $(69 \%)$. The three priority endemic zoonoses for the MOHs were leptospirosis, rabies, and brucellosis while the three priorities for the MAgs were brucellosis, rabies, and tuberculosis. Diagnosis for rabies and leptospirosis were cited as the capacities most in need of development. The most wanting cross-cutting capacity was coordination between stakeholders.

For emerging zoonoses, 28 ministries performed formal prioritization exercises. The top prioritization criteria were probability of introduction into the country and impact. The three priority emerging zoonoses for the MOHs were Ebola, avian influenza, and Chikungunya while for the MAgs were avian influenza, bovine spongiform encephalopathy (BSE) and West Nile Virus disease. Surveillance for avian influenza and Ebola, and diagnosis for BSE were quoted as the capacities most wanting.
For all zoonoses, the majority of respondents (68\%) ranked their relationship with the other Ministry as productive or very productive, and $31 \%$ minimally productive. Many countries demanded greater regional coordination, the constitution of a regional group to handle emerging zoonoses, and recommended the dissemination of reports on zoonoses occurrence and capacities across the region.

\section{Conclusions}

The survey is the first comprehensive effort to date to inform the status of zoonoses programs in LAC, and provides the evidence to build a regional strategy and identify capacity needs. A number of improvements appear evident: i) standardization of prioritization approaches, surveillance definitions and evaluation processes to support comparisons, ii) greater communication and coordination between countries, and iii) a platform to inform zoonoses occurrence in the region and the status of the region's capacities.

\section{Keywords}

zoonoses; prioritization; capacity building; survey; Pan American

\section{Acknowledgments}

Boren Fellowship.

\section{References}

1.Wang, LF, Crameri, G. Emerging zoonotic viral diseases. Rev Sci Tech Off Int Epiz. 2014; 33(569-81).

2.Schneider M.C. et al. Importance of animal/human health interface in potential Public Health Emergencies of International Concern in the Americas. Rev Panam Salud Publica. 2011; 29(371-9).

3.Hotez PJ. et al. The Neglected Tropical Diseases of Latin America and the Caribbean: A Review of Disease Burden and Distribution and a Roadmap for Control and Elimination. PLoS Negl Trop Dis. 2008; 2:e300.

*Melody J. Maxwell

E-mail: melody.j.maxwell@gmail.com 